\title{
Hybrid Spectral-IRDx: Near-IR and Ultrasound Attenuation System for Differentiating Breast Cancer from Adjacent Normal Tissue
}

\author{
Uttam M. Pal, Member, IEEE, Ashika Nayak*, Tejaswi Medisetti*, Gayatri Gogoi, Himanshu \\ Shekhar, M.S.N. Prasad, Jayant S. Vaidya, Hardik J. Pandya, Member, IEEE
}

\begin{abstract}
Objective: While performing surgical excision for breast cancer (lumpectomy), it is important to ensure a clear margin of normal tissue around the cancer to achieve complete resection. The current standard is histopathology; however, it is time-consuming and labour-intensive requiring skilled personnel. Method: We describe a Hybrid Spectral-IRDx - a combination of the previously reported Spectral-IRDx tool with multimodal ultrasound and NIR spectroscopy techniques. We show how this portable, cost-effective, minimal-contact tool could provide rapid diagnosis of cancer using formalin-fixed (FF) and deparaffinized (DP) breast biopsy tissues. Results: Using this new tool, measurements were performed on cancerous/fibroadenoma and its adjacent normal tissues from the same patients $(\mathrm{N}=14)$. The acoustic attenuation coefficient $(\alpha)$ and reduced scattering coefficient ( $\mu^{\prime}$ s) (at 850, 940, and $1060 \mathrm{~nm}$ ) for the cancerous/fibroadenoma tissues were reported to be higher compared to adjacent normal tissues, a basis of delineation. Comparing FF cancerous and adjacent normal tissue, the difference in $\mu$ 's at $850 \mathrm{~nm}$ and $940 \mathrm{~nm}$ were statistically significant ( $p=3.17 \mathrm{e}-2$ and $7.94 \mathrm{e}-3$ respectively). The difference in $\alpha$ between the cancerous and adjacent normal tissues for DP and FF tissues were also statistically significant $(p=2.85 \mathrm{e}-2$ and $7.94 \mathrm{e}-3$ respectively). Combining multimodal parameters $\alpha$ and $\mu$ 's (at 940 $\mathrm{nm})$ show highest statistical significance $(p=6.72 \mathrm{e}-4)$ between FF cancerous/fibroadenoma and adjacent normal tissues. Conclusion: We show that Hybrid Spectral-IRDx can accurately delineate between cancerous and adjacent normal breast biopsy tissue. Significance: The results obtained establish the proof-ofprinciple and large-scale testing of this multimodal breast cancer diagnostic platform for core biopsy diagnosis.
\end{abstract}

Index Terms-Near-infrared spectroscopy, Ultrasound attenuation, Breast cancer, Biopsy tissue, Label-free.

\section{INTRODUCTION}

B REAST cancer is the most common cancer in women. Breast cancers are found usually via a screening program or by the woman finding a breast lump, or other symptoms related to the breast. Diagnosis of breast lesions requires a triple

\footnotetext{
* Contributed equally.

Uttam M. Pal, Ashika Nayak, and Tejaswi Medisetti are with Department of Electronic Systems Engineering, the Indian Institute of Science, Bengaluru 560012, India.

Gayatri Gogoi is with Department of Pathology, Assam Medical College.

Himanshu Shekhar is an Assistant Professor at Department of Electrical Engineering, the Indian Institute of Technology Gandhinagar, Gandhinagar 382355, India.

M.S.N. Prasad is with Department of Pathology, The Bangalore Hospital, Bengaluru 560004, India.
}

assessment - with clinical history and examination, followed by imaging using X-Ray Mammography and/or Sonography [1], [2], and microscopic examination of tissue taken by a needlecore biopsy. The core biopsy is performed with the help of an ultrasound probe to localize the suspicious region so that a hollow needle can be used to collect a tissue sample. This core biopsy tissue is then usually fixed in formalin and then processed and embedded in paraffin blocks. These are then sliced to a few hundred micrometers, mounted on glass slides and stained using haematoxylin and eosin. These glass slides are examined by an expert histopathology doctor under a microscope to make a diagnosis of cancer. It is only after such confirmation that the treatment can be given by the clinical team- with surgery, radiotherapy, chemotherapy and biologic therapy [3]-[5].

Tissue diagnosis using histopathology is the gold-standard, but it is time-consuming, labour-intensive, and requires skilled personnel to perform the analysis. Although biopsy is necessary, it is often not available rapidly to assess adequacy of cancer resection in the operating room in resource-poor settings [3]-[5]. The clinician performing cancer margin assessment requires an immediate feedback over the tissue pathology to ensure cancer resection. The reported system can enhance the efficacy of cancer resection and optimize the workflow of cancer margin assessment.

Near-infrared (NIR) spectroscopy techniques have been proposed to delineate normal and cancerous tissues [2], [6][12]. Jacques et al. provide an extensive review of the optical properties for different types of tissues [9]. Pal et al. [2] proposed the Spectral-IRDx tool performing near-infrared spectroscopy while operating at $850 \mathrm{~nm}, 940 \mathrm{~nm}$, and $1060 \mathrm{~nm}$ to delineate cancer from adjacent normal deparaffinized breast biopsy tissues. Recent work by Schnell et al. [6] involves designing and developing a hybrid optical-infrared spectroscopy technique to visualize the structure and

Jayant S. Vaidya is Professor of Surgery and Oncology in Division of Surgery and Interventional Science, University College London, London WC1E 6BT, U.K.

Hardik J. Pandya is an Assistant Professor at Department of Electronic Systems Engineering, the Indian Institute of Science, Bengaluru 560012, India (correspondence email: hjpandya@iisc.ac.in). 

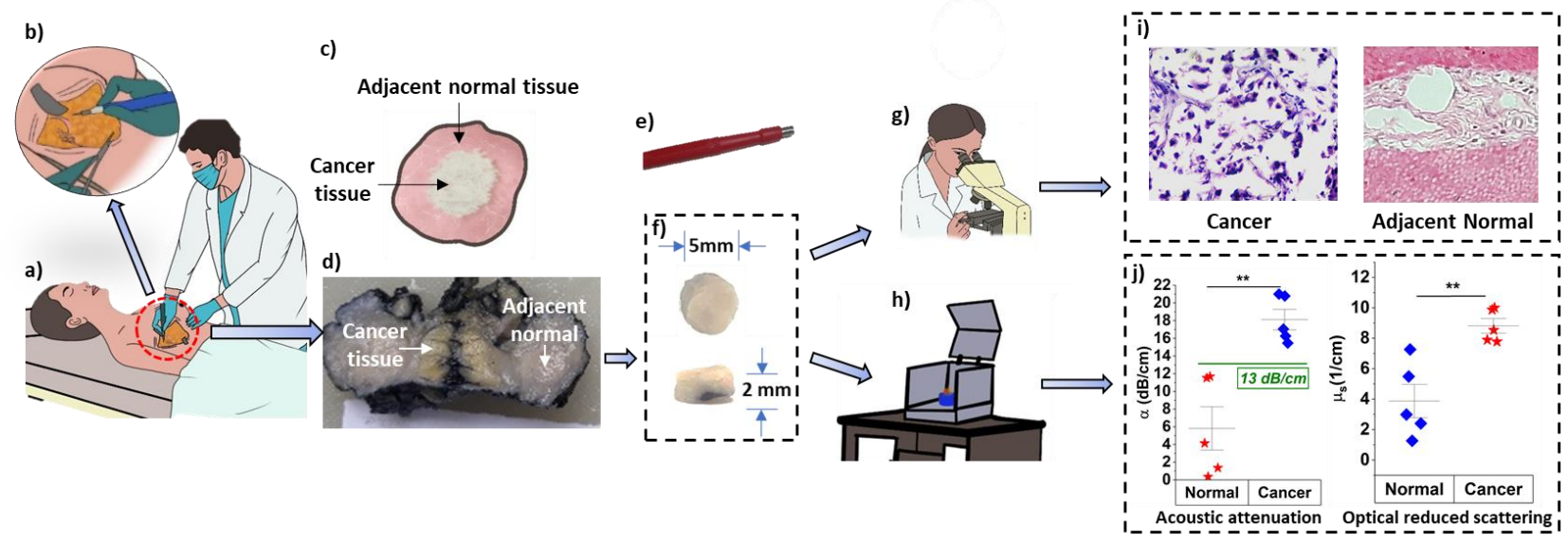

Fig. 1: Schematic representation of the standard histopathological method of breast cancer tissue diagnosis, in comparison with experimental assessment using Hybrid Spectral-IRDx. a) Clinician performing the breast lumpectomy surgery, b) Enlarged image at the excision procedure, c) Gross examination of cut surface, d) Excised breast tissue, f) Tissue core taken from the excised tissue used for experimental measurements using a punch biopsy instrument (e), g) Histopathologist examining stained slides, h) Taking readings with Hybrid Spectral-IRDx using multimodal CW Near-Infrared and TD Ultrasound, i) Histopathological image, and j) Readings from Hybrid Spectral-IRDx Tool.

composition of paraffinized tissue. However, further studies need to be performed on tissues that closely match the properties of fresh tissue. Commercial FTIR and Raman Spectroscopy tools such as Bruker's VERTEX 70v and Thermo Scientific's Smart Raman DXR have been proposed by Depciuch et al. [10], [11] and Fernandez [12] et al. respectively to characterize the bulk optical properties of the paraffinized breast biopsy tissue. However, such commercial tools are expensive and have not been designed or tested to evaluate millimeter-sized biopsy tissue samples. Anderson et al. proposed the continuous-wave near-infrared technique to quantify the tissue constituents such as haemoglobin, water, and lipid for the in-vivo cancerous tissue as compared to the adjacent normal. However, such systems needs to be designed and developed to perform a similar analysis for ex-vivo breast biopsy tissues.

High-frequency time-resolved ultrasound techniques have also been studied to diagnose cancer [13], [14]. Anastasiadis [13] reviewed high-frequency time-resolved ultrasound techniques to diagnose cancer. Ruby et al. [14] used the commercial Ultrasound system by SonixTouch to differentiate between malignant and benign breast tissue by quantifying the intrinsic reflection within the tissue using the Speed of Sound (SoS) technique. However, it is unclear whether such techniques are effective in differentiating tumor margins when using small (few $\mathrm{mm}$ ) ex vivo tissue samples. Moreover, further work needs to be performed to design point-based ultrasound measurement techniques for characterizing ex-vivo tissues.

Hybrid systems have been recently proposed combining the results from multiple techniques to give better accuracy in delineation. Contact MEMS-based techniques have been proposed that rely on characterizing the electrical, thermal, and mechanical properties of the tissues to differentiate healthy and cancerous tissue [15]-[18]. However, such contact-based techniques reduce the durability of the system. Alternative contact-less based technique include microwave-based imaging, which is has been performed on breast tissue- mimicking phantom tissues to delineate the diseased part of the tissue, albeit with lower sensitivity [19], [20].

The opto-acoustic multimodal system consisting of lightemitting diode and ultrasound transducer has been proposed [21]-[23] to diagnose cancer. Choi et al. [21] proposed the multimodal system to quantify the opto-acoustic properties of the eye tissues. Yuan et al. [22] performed the multimodal optoultrasound analysis on tissue-mimicking phantoms, however, further study needs to be performed on $e x$-vivo tissues. Choe et al. [23] performed the analysis on the treatment of the HeLa cells and its proliferation combining the light emitting diode and focused ultrasound measurements. The performance of this approach has not been assessed for bulk ex-vivo tissues.

Moreover, multimodal measurements reported previously have been performed in-vivo, which involves the acoustic fields propagating through multiple layers of normal and cancerous tissue. In the present study, we focus on measuring the bulk optical and acoustic properties of tissue from small ex-vivo sections. Testing of ex-vivo tissue circumvents confounding factors present in-vivo such as attenuation and beam propagation through several layers of tissue, which will likely have higher accuracy.

Low-cost systems have also been proposed to diagnose breast cancer [24]-[26]. Ghartey et al. [24] used Transillumination-based imaging; however, it requires manual visual intervention to distinguish breast cancer, which leads to lower accuracy and varying sensitivity. Min et al. [25] proposed Artificial Intelligence (AI) based Point-of-care system as an alternative to conventional microscopy to delineate cancerous cells. Vavadi et al. [26] designed a compact and cost-effective system based on combined ultrasound and diffuse optical imaging (DOI) and tested for tissue-mimicking phantoms. While these developments are encouraging, significant progress needs to be made before cost-effective, portable, and high accuracy systems can be realized for guiding tumor resection.

In this paper, we have upgraded the earlier reported SpectralIRDx Tool [2] with the ability to quantify the bulk acoustic and optical properties. Specifically, the Hybrid Spectral-IRDx 


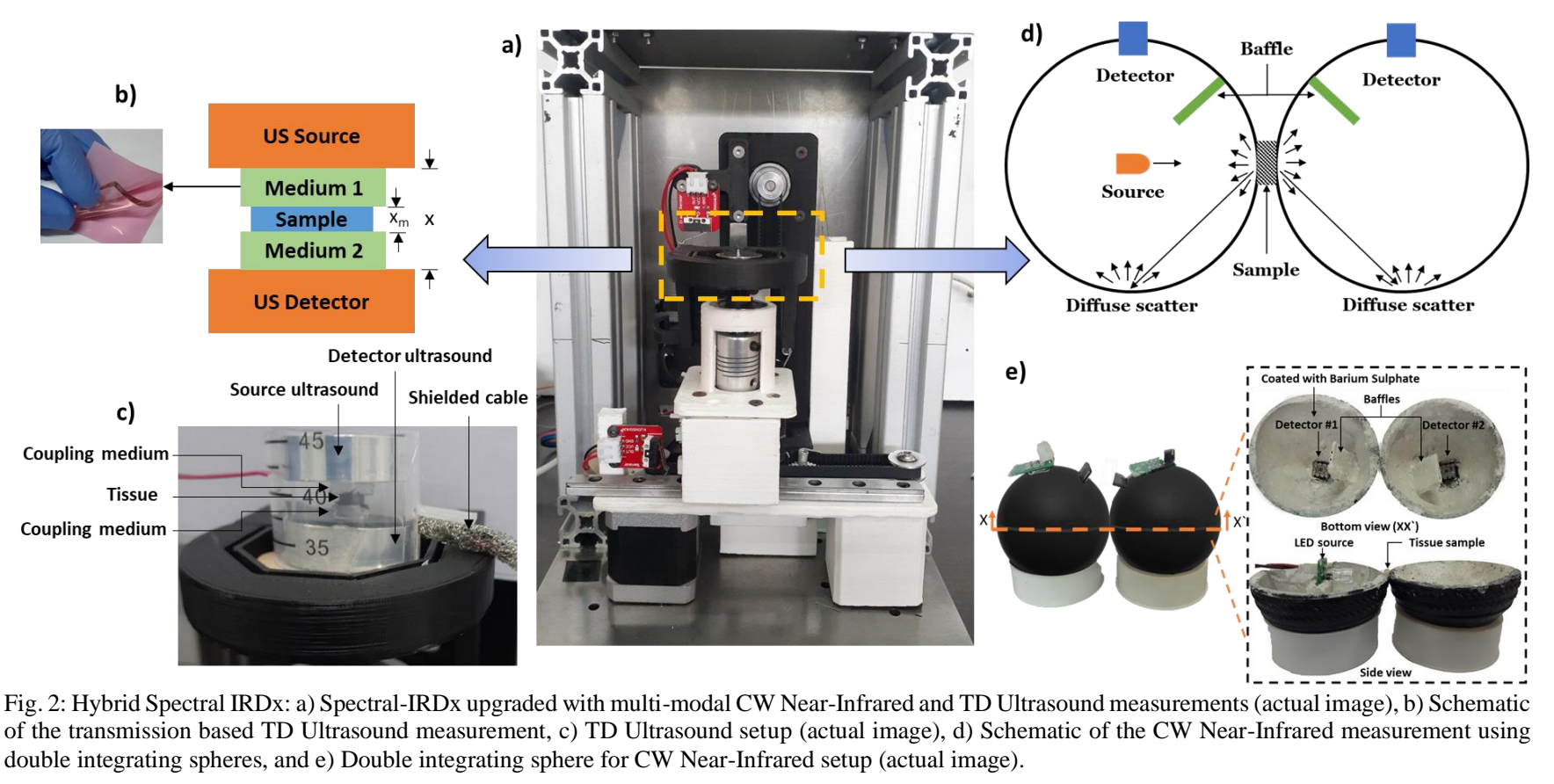

system can assess the acoustic attenuation coefficient $(\alpha)$, optical absorption coefficient $\left(\mu_{\mathrm{a}}\right)$, and reduced scattering coefficient ( $\mu_{\text {s }}$ ) of tissue. This system was used to characterize ex-vivo deparaffinized and formalin-fixed cancerous and adjacent normal tissues.

\section{Design And Methodology}

In this section, the sample preparation, theory, and instrumentation of the TD ultrasound and CW near-infrared spectroscopy techniques are discussed. The complete schematic of the histopathological method compared with the experimental assessment of the breast tissues for delineating cancer from adjacent normal is shown in Fig. 1.

\section{A. Sample Preparation}

During the breast excisional surgery, the surgeon typically removes cancerous tissue along with some of the adjacent normal tissue to ensure complete resection as shown in Fig.1 ad. These excised tissues undergo preservation through storage in either $10 \%$ formalin or paraffin-embedded blocks. In this study, the deparaffinized and formalin-fixed tissues were used to measure the tissues' bulk acoustic and optical properties. The sample preparation of the deparaffinized tissue is discussed in detail in our earlier work [2]. The preparation steps were further tailored from reported tissue sample preparation process of Baker et al. [27] with a consultant pathologist's help to make the tissue sample suitable for the NIR and ultrasound measurements. The core biopsy punch was used to manually conceive the tissue sample with thickness of $2 \mathrm{~mm}$ and diameter of $5 \mathrm{~mm}$ as shown in the Fig. 1e-f. The cancerous and adjacent normal tissue samples were identified with the help of consultant pathologist. The standard deviation of the tissue thickness of the deparaffinized and formalin-fixed tissue was $0.003 \mathrm{~mm}$ and $0.03 \mathrm{~mm}$, respectively.

The deparaffinized and formalin-fixed tissues have been reported to preserve the tissue constituents such as lipids and collagens [28], [29]. The same protocol for formalin-fixing and paraffinizing/deparaffinizing the tissue was used by the technician for all tissue samples. The bulk acoustic and optical properties of the fixed sample tissue may potentially vary between the patients. In this study we assume that this variation is small relative to the intrinsic differences between cancerous and adjacent normal tissue. Variation in the tissue properties, if any, due to differences in the fixing process is neglected in this study. All the studies were performed with written consent from patients through the institutional ethics committee of Assam Medical College and the Indian Institute of Science with the ethical clearance certificate number AM/EC/1333 and 1714012020 respectively.

\section{B. Time-Domain Ultrasound Attenuation Measurement Instrumentation (TD-UAM)}

The original Spectral-IRDx as reported in Pal et al. [2] performed near-infrared absorption spectroscopy, as shown in Fig. 2a. In the present work, the system was upgraded with detachable ultrasound and optical sub-system modules to characterize the bulk acoustic and optical properties respectively of the tissue.

Time-Domain ultrasound measurements was performed using the piezoelectric-based ultrasound transducer (PZT-5A) $(\mathrm{FWHM}=100 \mathrm{kHz})$ by Unictron Technologies (H2KLPY11000600) by using unipolar pulse as excitation. Such an excitation has been used previously for ultrasound imaging [30], [31]. The center frequency of the ultrasound transducer was $1 \mathrm{MHz}$, which is routinely used in biomedical applications [32], [33], and sufficient to resolve the tissue on length scales of few $\mathrm{mm}$. To reduce the impedance mismatch 
between the sample and acoustic transducer, a coupling medium (Unictron's proprietary gel pad) was used. The coupling medium is reusable and increases the durability of the ultrasound transducer, as the coupling medium avoids the direct contact of the transducer with the tissue sample. The tissue sample was placed between these gel pads as shown in Fig. 2 bc. As shown in Fig. 2b, the top ultrasound transducer is the source, and the bottom ultrasound transducer works as the detector. The ultrasound unipolar pulse signal generated by the source transducer traverses through the coupling gel pad, the tissue sample, the second coupling gel pad, and is finally received by the detector. Fig. $2 c$ showcases the actual experimental setup for the TD ultrasound measurements.

The ultrasound transducer was excited using unipolar pulse width with voltage excitation of $5 \mathrm{~V}$ (peak to peak) with a dc bias of $2.5 \mathrm{~V}$ through a function generator. The pulse width was varied from $200 \mathrm{~ns}$ to $1000 \mathrm{~ns}$ with a repetition frequency of 10 $\mathrm{kHz}$. The measured signal from the detector transducer was processed through a narrow-band pass filter tuned with a peak frequency of $1 \mathrm{MHz}$ to increase the signal to noise ratio (SNR) using high frequency OP2320 OPAMP. The output of the filter was recorded using a digital oscilloscope with a sampling rate of 20 mega samples per second.

\section{1) Ultrasound theoretical model}

To quantify the bulk acoustic attenuation coefficient, two cases were considered, one with the tissue sample and another with a reference sample. Considering the arrangement as shown in Fig. 2b, with the tissue sample, the detected voltage can be written as following [34]:

$$
V_{t}(f)=R_{t}(f) e^{-\alpha_{0} x} e^{-\left(\alpha-\alpha_{0}\right) x_{m}}(T)^{2}
$$

While with the reference medium instead of the tissue sample, the detected voltage can be written as:

$$
V_{r}(f)=R_{t}(f) e^{-\alpha_{0} x}
$$

where $V_{t}(f)$ and $V_{r}(f)$ are the detected voltage with tissue sample and reference medium respectively. $R_{t}(f)$ is the transfer function of the experimental system (electronics and ultrasound), $\mathrm{T}$ is the acoustic transmission coefficient at the tissue-medium interface. $\alpha_{0}$ and $\alpha$ are the bulk acoustic attenuation coefficient of the reference medium and tissue sample respectively, $x_{m}$ is the tissue sample thickness $(=2$ $\mathrm{mm})$, and $x$ is the distance between the ultrasound source and detector. The contribution of the attenuation constitutes of both absorption and scattering; however, the contribution of scattering in tissue attenuation is low and therefore only the absorption was considered [34]. By taking the ratio of Eqn. 1 over Eqn. 2, we get the following:

$$
\frac{V_{t}(f)}{V_{r}(f)}=e^{-\left(\alpha-\alpha_{0}\right) x_{m}}(T)^{2}
$$

Here, assuming the transmission coefficient $(\mathrm{T})$ at the interface of the coupling medium (i.e. gel pad) and tissue sample approaches unity, Eqn. 3 can be rewritten as the following:

$$
\frac{V_{t}(f)}{V_{r}(f)}=e^{-\left(\alpha-\alpha_{0}\right) x_{m}}
$$

Here, $V_{t}(f)$ and $V_{r}(f)$ are measured experimentally, $\alpha_{0}$ is assumed as $0.0022 \mathrm{~dB} / \mathrm{cm}, x_{m}$ is assumed as $2 \mathrm{~mm}$, and the bulk acoustic attenuation coefficient of the tissue sample $(\alpha)$ can be calculated from Eqn. 4.

Additionally, to quantify the variation between the normal and cancer tissue for different pulse widths, a new variable named Ultrasound Contrast Factor (UCF) was introduced as following:

$$
U C F\left(\frac{N}{C}\right)=\frac{\sum_{i=1}^{n_{s}}\left(\frac{N_{i}}{C_{i}}\right)}{n_{s}}
$$

where, $\mathrm{n}_{\mathrm{s}}=$ number of pairs of samples, $\mathrm{N}_{\mathrm{i}}$ = peak magnitude of adjacent normal tissue sample, and $\mathrm{C}_{\mathrm{i}}=$ peak magnitude of cancer or fibroadenoma tissue sample. The UCF quantifies the ratio of ultrasound attenuation of cancer and adjacent normal tissue for the pooled data.

\section{Continuous Wave Near-Infrared Spectroscopy Instrumentation (CW-NIRS).}

The instrumentation for the CW near-infrared spectroscopy involves three narrow beamwidth $\left(\sim 10^{\circ}\right)$ and narrow FWHM ( $40 \mathrm{~nm}$ ) light emitting diodes (LEDs) operating at three operating wavelengths $850 \mathrm{~nm}, 940 \mathrm{~nm}$, and $1060 \mathrm{~nm}$. The SFH4350 LED from OSRAM with a peak wavelength of 850 $\mathrm{nm}$, OFL-5102 LED from Multicomp with a peak wavelength of $940 \mathrm{~nm}$, and EOLD-1060-525 LED from OSA Optolight with a peak wavelength of $1060 \mathrm{~nm}$ are used. The operating wavelength of $850 \mathrm{~nm}$ was used for the higher absorption of collagen as compared to lipid, while the operating wavelength of $940 \mathrm{~nm}$ was used to nearly match the absorption peak of the lipid at $930 \mathrm{~nm}$, while the operating wavelength of $1060 \mathrm{~nm}$ results in almost the same absorption for the lipid and collagen [35], [36]. Fresnel lens was fixed in front of the LED to collimate the beam and focus at the center of the tissue sample.

The setup for the CW near-infrared measurement is showcased in Fig. 2 d-e. A double integrating sphere arrangement [37], [38] was used to quantify the bulk optical absorption coefficient $\left(\mu_{\mathrm{a}}\right)$ and bulk optical reduced scattering coefficient $\left(\mu_{\mathrm{s}}\right)$. The commercial integrating spheres are used to measure the bulk optical properties [39]-[41]; however, recently home-built integrating sphere is also being developed [42]. In our configuration, a rubber ball was cut open, and the LED source, detectors, baffle, and tissue sample were configured, as shown in Fig. 2e. The inner surface of the sphere was coated with Barium Sulphate, which was used as a reflectance standard with $98 \%$ reflectivity [39], [42]. The NIR source LED was kept at the center of the left sphere to increase the direct light tissue interaction, where the sample is placed at the exit port of the first integrating sphere. The detectors are attached at the top of each of the integrating spheres, while the baffle was used to reduce the direct light interaction from the sample to the detectors. To quantify the bulk optical absorption and reduced scattering coefficient, the measured reflectance 
$\left(\mathrm{M}_{\mathrm{R}}\right)$ and measured transmittance $\left(\mathrm{M}_{\mathrm{T}}\right)$ was quantified using the

following equations:

$$
\begin{gathered}
M_{R}=\frac{R(r, r, t, t)-R(0,0,0,0)}{R(r, r, 0,0)-R(0,0,0,0)} \\
M_{T}=\frac{T(r, r, t, t)-T(0,0,0,0)}{T(0,0,1,1)-T(0,0,0,0)}
\end{gathered}
$$

where, $\mathrm{R}(\mathrm{r}, \mathrm{r}, \mathrm{t}, \mathrm{t})$ and $\mathrm{T}(\mathrm{r}, \mathrm{r}, \mathrm{t}, \mathrm{t})$ are the detected voltages by detector \#1 and \#2 respectively in the setup as shown in Fig. 2d. $\mathrm{R}(\mathrm{r}, \mathrm{r}, 0,0)$ is the detected voltage from detector \#1, when a reflectance standard was placed at the exit port of the first integrating sphere. $\mathrm{T}(0,0,1,1)$ is the detected voltage by detector $\# 2$, when the sample was removed from the setup as shown in Fig. 2 d. $R(0,0,0,0)$ is the detected voltage by detector $\# 1$, when only the first integrating sphere was setup and the exit port of the sphere was kept open. $\mathrm{T}(0,0,0,0)$ is the detected voltage by detector \#2, when the LED is switched off. $R(0,0,0,0)$ and $\mathrm{T}(0,0,0,0)$ measure the noise which was removed from the actual measurements $\mathrm{R}(\mathrm{r}, \mathrm{r}, \mathrm{t}, \mathrm{t})$ and $\mathrm{T}(\mathrm{r}, \mathrm{r}, \mathrm{t}, \mathrm{t})$ respectively.

The Monte Carlo simulation is performed using the Inverse

TABLE I

\begin{tabular}{|c|c|}
\hline Requirements & Specification \\
\hline Power supply & $230 \mathrm{~V}(50 \mathrm{~Hz})$ \\
\hline System dimensions & $\begin{array}{l}20.3 \mathrm{~cm} \text { (length) x } 18 \mathrm{~cm} \text { (breadth) } \\
\text { x } 26.8 \mathrm{~cm} \text { (height) }\end{array}$ \\
\hline System weight & $5.5 \mathrm{Kg}$ \\
\hline $\begin{array}{l}\text { Ultrasound operation } \\
\text { type }\end{array}$ & Time domain (TD) \\
\hline Ultrasound transducer & $\begin{array}{l}\text { PZT-5A based Piezoelectric } \\
\text { transducer (H2KLPY11000600) }\end{array}$ \\
\hline Optical operation type & Continuous wave $(\mathrm{CW})$ \\
\hline Operating wavelength & $850 \mathrm{~nm}, 940 \mathrm{~nm}$, and $1060 \mathrm{~nm}$ \\
\hline Optical source & $\begin{array}{l}850 \mathrm{~nm} \text { (SFH4350), } 940 \mathrm{~nm} \text { (OFL- } \\
5102) \text {, and } 1060 \mathrm{~nm} \text { (EOLD-1060- } \\
525)\end{array}$ \\
\hline Optical detector & $\begin{array}{l}\text { Si PD with integrated amplifier } \\
\text { (OPT101) }\end{array}$ \\
\hline Portable & Yes \\
\hline Tissue sample dimension & $5 \mathrm{~mm}$ diameter and $2 \mathrm{~mm}$ thickness \\
\hline Tissue type & $\begin{array}{l}\text { Deparaffinized FFPE and Formalin- } \\
\text { Fixed tissue samples }\end{array}$ \\
\hline $\begin{array}{l}\text { Approximate cost of the } \\
\text { prototype }\end{array}$ & $\$ 650$ \\
\hline
\end{tabular}

HYBRID SPECTRAL-IRDX SYSTEM SPECIFICATION

Adding-Doubling software to quantify the bulk optical absorption and scattering coefficient. The input required by the software includes $M_{R}, M_{T}$, the thickness of the sample, reflectivity of reflectance standard, number of spheres, port dimensions, the refractive index of tissue sample and the diameter of illumination beam [37]. The complete specification, including the optical and ultrasound configuration, is summarized in Table I.

\section{Measurement of Human Tissue Samples}

The measurements were performed on tissue samples from $\mathrm{N}=$ 14 patients. The tissue samples from $\mathrm{N}=4$ patients were preserved using FFPE (Formalin-fixed Paraffin-embedded) and the tissues of the remaining $\mathrm{N}=10$ patients were preserved using $10 \%$ formalin solution. A total of $\mathrm{n}=28$ core biopsy tissue samples were obtained from 14 patients. Time-Domain
Ultrasound Attenuation Measurement (TD-UAM) was performed on both the deparaffinized and formalin-fixed tissue. Continuous-Wave Near-Infrared Spectroscopy (CW-NIRS) was performed only on formalin-fixed tissue, because CWNIRS analysis on deparaffinized tissue was performed in our earlier work [2]. The tissue type along with the pathology and measurement technique are summarized in Table II. The nonparametric Mann Whitney U Test was performed to quantify the statistical difference between the cancerous and adjacent normal tissues of individual techniques. While the Fisher's combined probability test was used to perform statistical analysis of the combined ultrasound and optical measurements. Both the near-infrared and ultrasound measurements were carried out three times, and the average values were considered to perform the statistical analysis.

TABLE II

\begin{tabular}{|c|c|c|c|c|c|c|}
\hline $\begin{array}{c}\text { Type of } \\
\text { tissue } \\
\text { samples }\end{array}$ & \multicolumn{2}{|c|}{$\begin{array}{c}\text { De- } \\
\text { paraffinized } \\
(\mathrm{N}=4)\end{array}$} & \multicolumn{2}{|c|}{$\begin{array}{c}\text { Formalin- } \\
\text { fixed } \\
(\mathbf{N}=\mathbf{5})\end{array}$} & \multicolumn{2}{|c|}{$\begin{array}{c}\text { Formalin- } \\
\text { fixed } \\
(\mathbf{N}=5)\end{array}$} \\
\hline $\begin{array}{c}\text { Tissue } \\
\text { pathology }\end{array}$ & IDC & $\mathbf{A N}$ & IDC & AN & FB & AN \\
\hline $\begin{array}{c}\text { \# of tissue } \\
\text { samples }\end{array}$ & $n=4$ & $n=4$ & $n=5$ & $n=5$ & $n=5$ & $n=5$ \\
\hline $\begin{array}{c}\text { Measure- } \\
\text { ment } \\
\text { techniques }\end{array}$ & \multicolumn{2}{|c|}{ TD-UAM } & \multicolumn{2}{|c|}{$\begin{array}{l}\text { TD- UAM and } \\
\text { CW-NIRS }\end{array}$} & \multicolumn{2}{|c|}{$\begin{array}{l}\text { TD-UAM } \\
\text { and CW- } \\
\text { NIRS }\end{array}$} \\
\hline
\end{tabular}

DISTRIBUTION OF TISSUE PATHOLOGY

$\mathrm{N}$ is the number of patients

$n$ is the number of tissue samples.

IDC is Invasive Ductal Carcinoma,

AN is Adjacent Normal,

FB is Fibroadenoma.

TD-UAM is Time-Domain Ultrasound Attenuation Measurement, and CW-NIRS is Continuous-Wave Near-Infrared Spectroscopy.

\section{RESULtS AND DISCUSSION}

\section{A. Near-Infrared and Acoustic Attenuation Results.}

The Time-Domain (TD) ultrasound response signal for deparaffinized (DP) and formalin-fixed (FF) tissue generated by varying the pulsewidth between $200 \mathrm{~ns}$ and $1000 \mathrm{~ns}$ is shown in Fig 3a-d respectively. The Ultrasound Contrast Factor (UCF) for both the DP and FF tissue peaked at pulse width of $500 \mathrm{~ns}$ with values 2.66 and 14.57 respectively as shown in the Fig. 3ab. The reason for the highest differentiation at $500 \mathrm{~ns}$ could be due to insufficient energy at lower pulsewidth, while the excitation ceases to be an impulse for higher pulse width. The experimentally measured Time-Domain response of DP and FF tissue sample for $500 \mathrm{~ns}$ pulsewidth was smoothened with 200 points and reported in Fig. 3c-d respectively. It can be observed that for the fibroadenoma and cancerous tissues, the peak magnitude of the response envelope reduces drastically as compared to the TD response from adjacent normal tissues.

The large inter-patient variation in the magnitude of the envelope of the time domain ultrasound response for the adjacent normal tissues was observed. The primary reason behind these large variations was due to the inter-patient variation in the density of the breast tissue [43]. The other factors affecting the density of the breast include whether the woman is under follicular or luteal phase of her menstrual cycle 

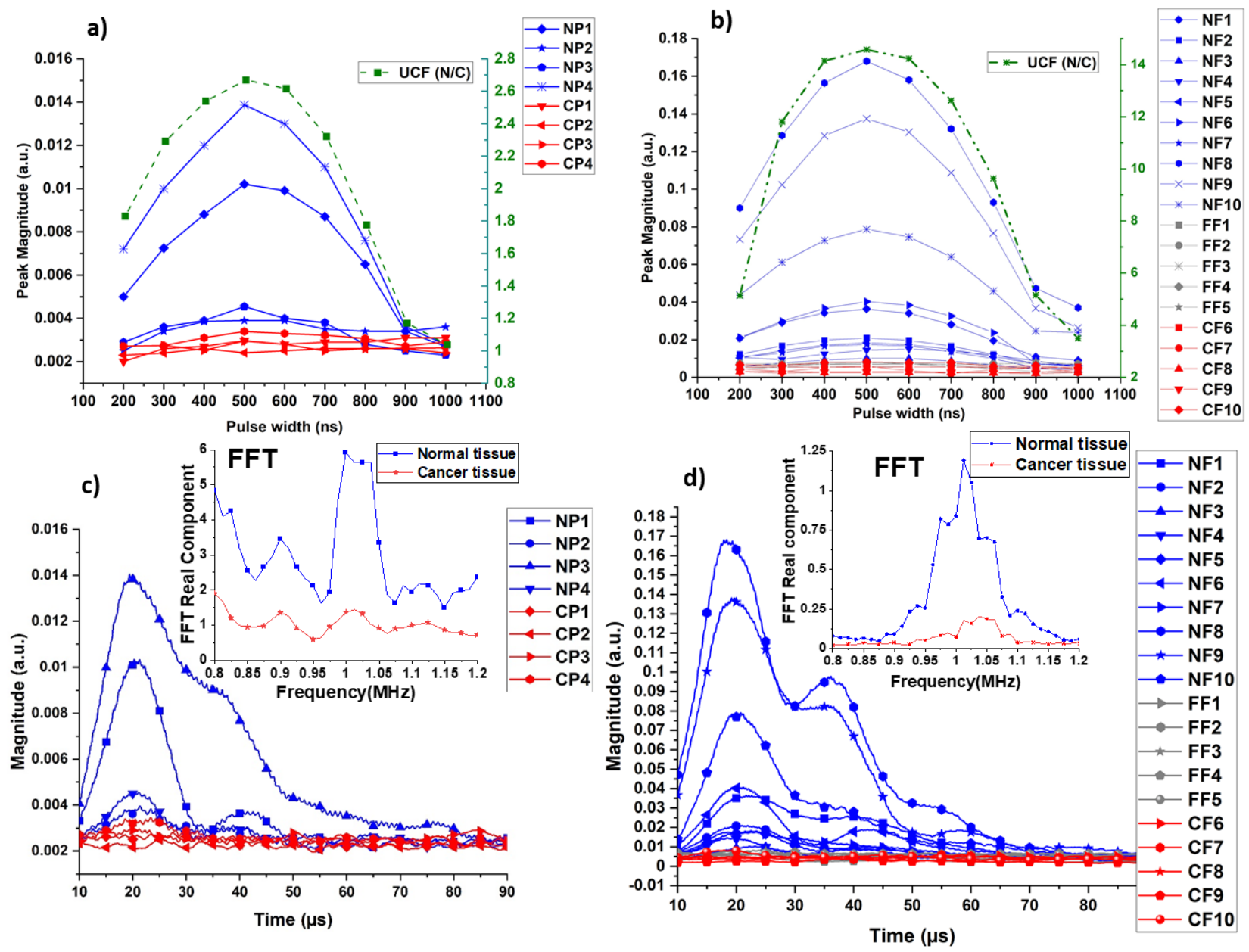

Fig. 3. Peak magnitude of ultrasound time-domain response vs. pulse width analysis: a) deparaffinized and b) formalin fixed tissue. Time-domain Ultrasound response with pulse width of $500 \mathrm{~ns}$ for c) $\mathrm{N}=8$ deparaffinized along with FFT analysis of a single adjacent normal and cancer response (inset) and d) $\mathrm{N}=10$ formalin-fixed tissue along with FFT Analysis (inset). NP and CP are the abbreviations for adjacent normal and cancer deparaffinized tissue respectively, while NF, FF, and CF are the abbreviations for adjacent normal, fibroadenoma, and cancerous formalin-fixed tissues respectively.

[44], attained menopause [45], age [46], and BMI [47].

The pooled data results for the bulk acoustic attenuation $(\alpha)$ coefficient and the bulk optical absorption $\left(\mu_{\mathrm{a}}\right)$ and reduced scattering coefficient $\left(\mu_{s}^{\prime}\right)$ are shown in Fig. 4. From the acoustic attenuation coefficient, Fig. $4 a$, it can be inferred that TD ultrasound response for both DP (cancerous and adjacent normal) and $\mathrm{FF}$ (cancerous/fibroadenoma and adjacent normal) tissue are statistically significant $(p=2.8 \mathrm{e}-2,7.94 \mathrm{e}-3$, and $7.94 \mathrm{e}-3$ respectively). The acoustic attenuation coefficient for FF tissue showed a better delineation ( $p=7.94 \mathrm{e}-3)$ than the DP tissue ( $p=0.028$ ), likely due to better preservation of the tissue properties. The difference in mean acoustic attenuation coefficient between FF cancerous and adjacent normal tissue is higher $(3.78 \mathrm{~dB} / \mathrm{cm})$ compared to the FF fibroadenoma and adjacent normal tissues $(1.13 \mathrm{~dB} / \mathrm{cm})$, a basis of delineation between fibroadenoma and cancerous tissue. The mean acoustic attenuation coefficient for the cancerous and adjacent normal tissue for the DP tissue was $20.62 \mathrm{~dB} / \mathrm{cm}$ and $16.18 \mathrm{~dB} / \mathrm{cm}$ respectively. The mean acoustic attenuation coefficient for the cancerous and adjacent normal tissue for the FF tissues are $18.11 \mathrm{~dB} / \mathrm{cm}$ and $5.80 \mathrm{~dB} / \mathrm{cm}$ respectively. The results are in accordance with the previous findings of higher acoustic attenuation in the DP and FF tissue than fresh tissue [34], [48] caused by some degradation in the structural properties during processing. Additionally, the results are along the lines of several literature that report higher acoustic attenuation coefficient in cancerous tissue than normal tissues [48]-[50]. The acoustic attenuation coefficient for the FF and DP tissues are tabulated in Table III and IV respectively.

The difference in optical reduced scattering coefficient $\left(\mu^{\prime}{ }_{s}\right)$ between FF cancerous and adjacent normal tissues were reported to be statistically significant for $850 \mathrm{~nm}(p=3.17 \mathrm{e}-2)$, $940 \mathrm{~nm}(p=7.94 \mathrm{e}-3)$, and $1060 \mathrm{~nm}(p=3.17 \mathrm{e}-2)$ as shown in Fig. 4b. However, the difference in $\mu$ 's between FF fibroadenoma and adjacent normal tissues were reported to be statistically significant only for $940 \mathrm{~nm}(p=7.94 \mathrm{e}-3)$. The mean value of $\mu_{\text {s }}^{\prime}$ at $850 \mathrm{~nm}, 940 \mathrm{~nm}$, and $1060 \mathrm{~nm}$ for the FF cancerous tissues $(14.591 / \mathrm{cm}, 8.811 / \mathrm{cm}$, and $5.571 / \mathrm{cm})$ were 


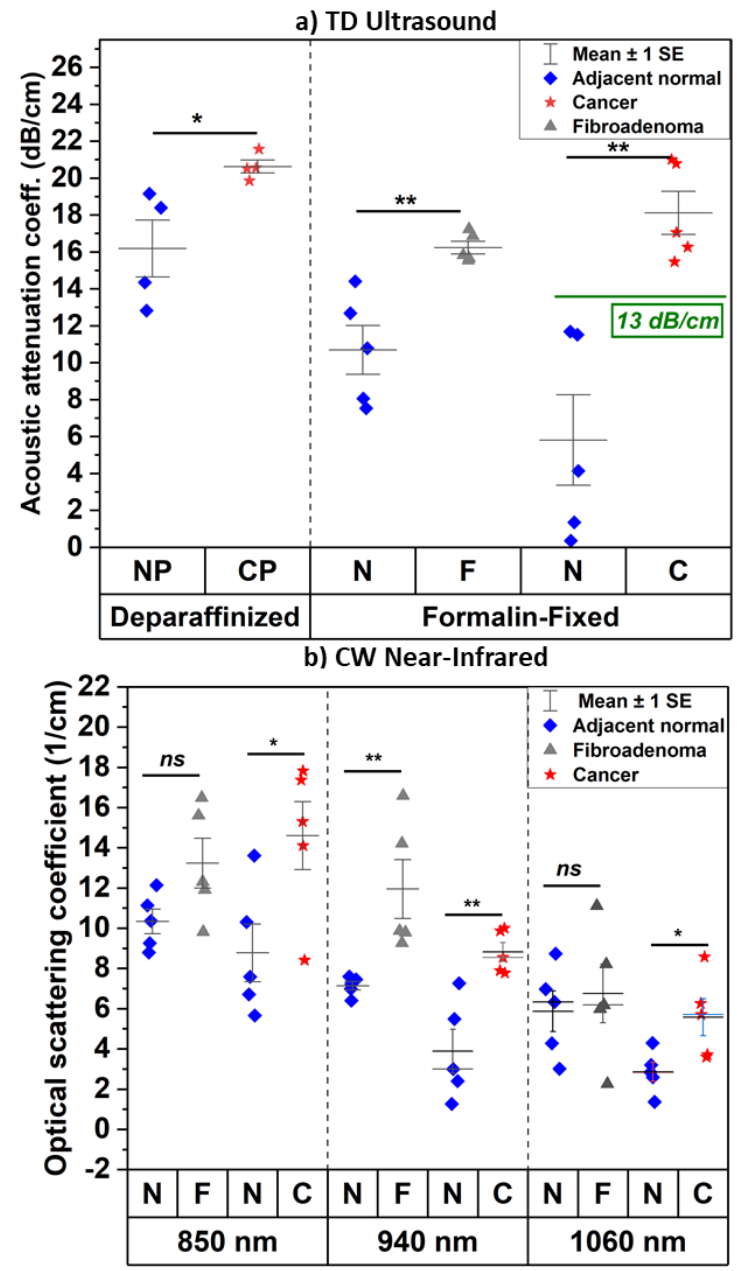

Fig. 4. Pooled data for Hybrid Spectral-IRDx measurement with scatter plots for: a) Acoustic attenuation measured with excitation pulse width of $500 \mathrm{~ns}$, and b) Optical reduced scattering coefficient for CW NIR measurement. The green lines and the adjacent values show the cut-off that can separate cancer from adjacent normal tissues.

reported to be higher compared to the FF adjacent normal tissue $(8.871 / \mathrm{cm}, 3.881 / \mathrm{cm}$, and $2.851 / \mathrm{cm})$. The findings agree with the data reported in the literature where a greater reduced scattering coefficient in cancer is found compared to normal tissue [51], with around 20\% increase as reported in Fang et al. [52] and Grosenick et al. [53], while $50 \%$ increase as reported in Choe et al. [54]. In this work, we report an average increase of nearly $66 \%$ and $120 \%$ in the $\mu^{\prime}$ s in the cancerous tissues compared to the adjacent normal tissues at $850 \mathrm{~nm}$ and $940 \mathrm{~nm}$ respectively.

The mean value of $\mu^{\prime}{ }_{s}$ at $940 \mathrm{~nm}$ for the FF fibroadenoma tissues $(11.941 / \mathrm{cm})$ was higher the FF cancerous tissues $(8.81$ $1 / \mathrm{cm})$.

The mean optical absorption coefficient $\left(\mu_{\mathrm{a}}\right)$ for adjacent normal tissues were reported to be higher $(2.041 / \mathrm{cm}, 3.20$ $1 / \mathrm{cm}$, and $3.671 / \mathrm{cm})$ compared to the cancerous tissues $(1.12$ $1 / \mathrm{cm}, 1.511 / \mathrm{cm}$, and $1.941 / \mathrm{cm}$ ) at $850 \mathrm{~nm}, 940 \mathrm{~nm}$, and 1060 $\mathrm{nm}$ respectively; however none were reported to be statistically different. The absolute values of $\mu_{\mathrm{a}}$ and $\mu^{\prime}$ s fall in the range of the recently reported literature [7], [52]-[54]. The $\mu_{\mathrm{a}}$ and $\mu^{\prime}{ }_{\mathrm{s}}$ for the formalin-fixed tissues are tabulated in Table III.

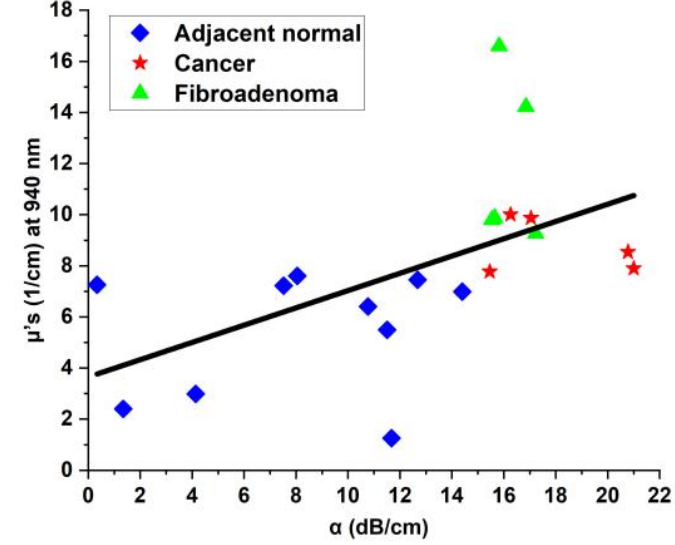

Fig. 5. Correlation between acoustic attenuation coefficient $(\alpha)$ and optical reduced scattering coefficient $\left(\mu_{\mathrm{s}}^{\prime}\right)$ at $940 \mathrm{~nm}$ for the adjacent normal, fibroadenoma, and cancerous tissues.

\section{B. Analysis on the combination of Results from Optical and Ultrasound Measurements}

The Fisher's combined probability test was used to perform combined p-value test over the independent ultrasound and optical measurements. When the ultrasound results were combined with the optical, the probability of delineating the cancerous/fibroadenoma from the adjacent normal tissue increased further. Specifically, the difference between the FF cancerous and adjacent normal tissues achieved the highest statistical significance when the results of $\alpha$ and $\mu^{\prime}$ s (at $940 \mathrm{~nm}$ ) were combined ( $p=6.72 \mathrm{e}-4)$. Combining the results of $\alpha$ and $\mu_{\text {s }}$ (at $850 \mathrm{~nm}, 940 \mathrm{~nm}$, and $1060 \mathrm{~nm}$ ) for FF tissues also reported high statistical significance $(p=9.53 \mathrm{e}-4)$. The difference between the FF fibroadenoma and adjacent normal tissues achieve the highest statistical significance when the results of $\alpha$ and $\mu$ 's (at $940 \mathrm{~nm}$ ) were combined ( $p=6.72 \mathrm{e}-4$ ). The $\mathrm{p}$-value for each of the individual and the combined tests are tabulated in Table $\mathrm{V}$. The correlation between the ultrasound attenuation coefficient and optical scattering coefficient is shown in Fig. 5. It can be noted that the measurements for the diseased (Fibroadenoma and carcinoma) tissues were observed to be in the top-right quadrant, a basis of delineation of the combined measurements. Furthermore, the ultrasound attenuation coefficient accounts for limited variation $\left(\mathrm{R}^{2}=0.268\right)$ in the optical scattering coefficient. This also means that each of the independent variable ( $\mu$ 's and $\alpha$ ) add new information regarding the tissue property.

\section{Calibration and Cut-offs for the system}

The readings using this tool were particularly remarkable because we could see a clear separation between all the examined cancer and normal tissues for $\alpha$. Therefore, based on the observed results, we proposed a reasonable cut-off of 13 $\mathrm{dB} / \mathrm{cm}$ for the acoustic attenuation coefficient $(\alpha)$, specifically for the formalin-fixed tissues. We plan to test the validity of the cut-offs in the next project with a much larger sample size.

\section{Discussion on the Physiology of Cancer and Normal} Tissue

The primary mechanism of the light scattering in biological tissue is due to Mie scattering, which is related to the size and 
TABLE III

BULK ACOUSTIC AND OPTICAL PROPERTIES FOR FORMALIN FIXED TISSUES

\begin{tabular}{|c|c|c|c|c|c|c|c|c|c|c|c|c|c|c|}
\hline SN & \multicolumn{2}{|c|}{$\alpha(\mathrm{dB} / \mathrm{cm})$} & \multicolumn{6}{|c|}{$\mu_{\mathrm{s}}^{\prime}(1 / \mathrm{cm})$} & \multicolumn{6}{|c|}{$\mu_{\mathrm{a}}(1 / \mathrm{cm})$} \\
\hline & & & & & & nm & 106 & $\mathbf{n m}$ & & & 940 & & 106 & $\mathrm{~nm}$ \\
\hline \multicolumn{15}{|c|}{ Comparison of fibroadenoma with adjacent normal tissues } \\
\hline & $\mathbf{N}$ & $\mathbf{F}$ & $\mathbf{N}$ & $\mathbf{F}$ & $\mathbf{N}$ & $\mathbf{F}$ & $\mathbf{N}$ & $\mathbf{F}$ & $\mathbf{N}$ & $\mathbf{F}$ & $\mathbf{N}$ & $\mathbf{F}$ & $\mathbf{N}$ & $\mathbf{F}$ \\
\hline 1 & 8.05 & 16.85 & 8.79 & 9.82 & 7.60 & 14.22 & 6.978 & 11.12 & 1.20 & 1.35 & 1.787 & 0.46 & 0.79 & 2.88 \\
\hline 2 & 10.77 & 15.82 & 10.36 & 16.46 & 6.41 & 16.59 & 8.736 & 8.21 & 3.59 & 1.53 & 0.85 & 0.88 & 4.073 & 2.34 \\
\hline 3 & 14.40 & 15.54 & 9.26 & 15.61 & 6.99 & 9.79 & 4.283 & 6.18 & 0.96 & 0.87 & 3.19 & 0.72 & 0.14 & 0.50 \\
\hline 4 & 12.68 & 15.65 & 12.14 & 12.31 & 7.45 & 9.87 & 6.335 & 5.98 & 1.38 & 0.64 & 0.73 & 0.32 & 3.96 & 0.32 \\
\hline 5 & 7.522 & 17.23 & 11.14 & 11.92 & 7.22 & 9.26 & 3.012 & 2.26 & 0.82 & 0.55 & 0.54 & 0.45 & 0.26 & 0.72 \\
\hline Avg. & 10.68 & 16.21 & 10.33 & 13.22 & 7.13 & $\mathbf{1 1 . 9 4}$ & 5.86 & 6.75 & 1.59 & 0.98 & 1.41 & 0.56 & 1.84 & 1.35 \\
\hline \multicolumn{15}{|c|}{ Comparison of cancerous with adjacent normal tissues } \\
\hline & $\mathbf{N}$ & $\mathbf{C}$ & $\mathbf{N}$ & $\mathbf{C}$ & $\mathbf{N}$ & $\mathbf{C}$ & $\mathbf{N}$ & C & $\mathbf{N}$ & $\mathbf{C}$ & $\mathbf{N}$ & $\mathbf{C}$ & $\mathbf{N}$ & $\mathbf{C}$ \\
\hline 6 & 11.51 & 16.26 & 6.70 & 15.3 & 5.49 & 10.0 & 2.593 & 3.71 & 0.39 & 1.28 & 3.68 & 0.32 & 4.33 & 0.36 \\
\hline 7 & 11.68 & 17.05 & 10.30 & 14.10 & 1.26 & 9.87 & 2.84 & 3.60 & 2.39 & 0.48 & 3.66 & 0.41 & 3.94 & 2.48 \\
\hline 8 & 0.347 & 21.00 & 13.61 & 17.82 & 7.26 & 7.89 & 4.29 & 6.26 & 1.04 & 1.19 & 3.56 & 2.94 & 4.45 & 3.12 \\
\hline 9 & 1.348 & 20.78 & 5.66 & 8.41 & 2.40 & 8.54 & 1.37 & 8.58 & 3.57 & 1.99 & 3.68 & 1.88 & 3.29 & 2.57 \\
\hline 10 & 4.140 & 15.46 & 7.59 & 17.36 & 2.99 & 7.77 & 3.20 & 5.71 & 2.84 & 0.69 & 1.44 & 2.00 & 2.36 & 1.21 \\
\hline Avg. & $\begin{array}{l}5.80 \\
\end{array}$ & 18.11 & 8.77 & 14.59 & 3.88 & 8.81 & 2.85 & 5.57 & 2.04 & 1.12 & 3.20 & 1.51 & 3.67 & 1.94 \\
\hline
\end{tabular}

$\alpha-$ Acoustic attenuation coefficient $(\mathrm{dB} / \mathrm{cm})$.

$\mu_{\mathrm{s}}^{\prime}-$ Optical reduced scattering coefficient $(1 / \mathrm{cm})$

$\mu_{\mathrm{a}}-$ Optical absorption coefficient $(1 / \mathrm{cm})$.

$\mathrm{F}$ - Formalin-fixed fibroadenoma tissues

$\mathrm{N}$ - Formalin-fixed adjacent normal tissues

$\mathrm{C}$ - Formalin-fixed cancer tissues

TABLE IV

BULK ACOUSTIC PROPERTIES FOR DEPARAFFINIZED TISSUES

\begin{tabular}{|l|c|c|}
\hline SN & \multicolumn{2}{|c|}{$\boldsymbol{\alpha}(\mathbf{d B} / \mathbf{c m})$} \\
\hline & $\mathbf{N P}$ & $\mathbf{C P}$ \\
\hline 1 & 14.352 & 20.51 \\
\hline 2 & 19.15 & 21.57 \\
\hline 3 & 12.81 & 20.54 \\
\hline 4 & 18.39 & 19.85 \\
\hline Avg. & $\mathbf{1 6 . 1 8}$ & $\mathbf{2 0 . 6 2}$ \\
\hline
\end{tabular}

$\mathrm{CP}$ - Deparaffinized cancerous tissues

NP - Deparaffinized adjacent normal tissues

density of the biological cells, tumour cell proliferation and infiltration, and the anisotropic arrangement of tissue constituents such as collagen fibrils. These structural changes cause inhomogeneity, and hence, the light propagating through the tissue undergoes multiple gradients and discontinuities of refractive indexes, causing higher optical reduced scattering coefficient in cancer and fibroadenoma tissues as compared to adjacent normal tissues [7], [55]. The optical absorption coefficient relates to the absorption spectra of the different constituents of tissues. Higher optical absorption at $940 \mathrm{~nm}$ in normal tissues as compared to cancer indicates the correlation with the higher absorption peak of the lipids. This indicates a higher concentration of lipids in normal as compared to cancer, as reported in the literature [2], [7], [8], [56]. The cancerous tissue undergoing desmoplastic reaction leads to higher fibrous and connective tissues. This phenomenon causes higher reflection and attenuation by cancerous and fibroadenoma tissue than the adjacent normal tissues [49], [50], leading to a higher attenuation coefficient in cancerous tissue compared to normal. However, further research needs to be performed to better understand the bulk acoustic property of the tissue.

\section{E. Limitation of the Study and Future Work}

One limitation of the study is the small sample size. Although the tool is a proof-of-principle of using a combination of these
TABLE V

STATISTICAL ANALYSIS OF COMBINED PARAMETERS FOR CANCEROUS FORMALIN-FIXED (FF) TISSUES

\begin{tabular}{|c|c|c|}
\hline Parameters & $C \& N$ & F\& $N$ \\
\hline$\alpha$ & $7.94 \mathrm{e}-3(* *)$ & $7.94 \mathrm{e}-3(* *)$ \\
\hline$\mu_{\mathrm{s}}^{\prime}(850 \mathrm{~nm})$ & $3.17 \mathrm{e}-2(*)$ & $9.52 \mathrm{e}-2(*)$ \\
\hline$\mu_{\mathrm{s}}(940 \mathrm{~nm})$ & 7.94 e-3 (**) & 7.94 e-3 $(* *)$ \\
\hline$\mu_{\text {'s }}(1060 \mathrm{~nm})$ & $3.17 \mathrm{e}-2(*)$ & $\mathrm{ns}$ \\
\hline$\mu_{\mathrm{s}}^{\prime}(850 \mathrm{~nm}, 940 \mathrm{~nm}, \& 1060 \mathrm{~nm})$ & $1.09 \mathrm{e}-2(*)$ & $6.18 \mathrm{e}-3(* *))^{\#}$ \\
\hline$\alpha$ and $\mu_{\mathrm{s}}(940 \mathrm{~nm})$ & $6.72 \mathrm{e}-4(* * *)$ & $6.72 \mathrm{e}-4(* * *)$ \\
\hline$\alpha, \mu_{\mathrm{s}}^{\prime}(850 \mathrm{~nm})$, and $\mu_{\mathrm{s}}^{\prime}(940 \mathrm{~nm})$ & $1.09 \mathrm{e}-2(*)$ & $2.57 \mathrm{e}-2(*)$ \\
\hline $\begin{array}{c}\alpha, \mu_{\mathrm{s}}^{\prime}(850 \mathrm{~nm}), \mu^{\prime}(940 \mathrm{~nm}), \text { and } \\
\mu_{\mathrm{s}}^{\prime}(1060 \mathrm{~nm})\end{array}$ & $9.53 \mathrm{e}-4(* * *)$ & $2.57 \mathrm{e}-2(*)^{\#}$ \\
\hline
\end{tabular}

approaches to achieve high accuracy, it needs to be validated in a larger number of samples. Secondly, for these experiments we used deparaffinized and formalin-fixed tissues. The tool would be most useful if used on fresh tissue. Human tissue changes its physical properties with tissue-fixing processes, reducing some of the physical properties, for example, when fresh, normal tissue is soft and cancer hard, and this difference is to some extent lost after formalin-fixation. Therefore, we believe that the accuracy may be even better with fresh tissue because of the preserved original physical properties.

As part of our further studies, we envisage to perform further experiments with a large sample size and test the tool on fresh tissues. In addition, we intend to perform inter-lesion variability study by analysis of the ultrasound and optical parameters across different pathologies of cancer. We will also use highfrequency broadband polyvinylidene fluoride (PVDF)-based ultrasound transducer to characterize broadband spectrum tissue acoustic response, and time-domain optical response to quantify the optical absorption and reduced scattering coefficient. In future, we plan to also develop a probe integrating NIR and piezoelectric based ultrasound array to test the in-vivo breast tissue. 


\section{COST ANALYSIS}

The entire cost of the prototype including the PZT based ultrasound transducer, source LED, detector OPT101, electronic components, function generator, digital oscilloscope, mechanical components, fabrication, and display system amounts to $\sim \$ 650$.

\section{CONCLUSION}

In this paper, we demonstrate the feasibility of using combination of multimodal ultrasound and near-infrared measurements to delineate ex-vivo cancerous tissue from adjacent normal tissue. For the acoustic attenuation coefficient $(\alpha)$, the highest accuracy was achieved with a pulse width of $500 \mathrm{~ns}$. For optical reduced scattering coefficient $\left(\mu^{\prime}{ }_{s}\right)$, the best accuracy was obtained at $940 \mathrm{~nm}$. However, the delineation with the highest statistical significance was observed when the results of $\alpha$ (at $500 \mathrm{~ns}$ pulse width) was combined with $\mu_{\text {'s }}$ (at $940 \mathrm{~nm})$.

This novel Hybrid-Spectral-IRDx is designed to be portable, minimal-contact, robust, and easy-to-interpret. If the accuracy of Hybrid-Spectral-IRDx is found to be approaching that of the histopathological examination in larger studies, then this approach has a great potential for being widely used in the care of cancer patients.

\section{AUTHORS' CONTRIBUTIONS}

Conceptualization: UP and HJP

Tool design and fabrication: UP and HJP

Experiments: UP and HJP

Analysis: UP, AN, TM, HS, JSV, and HJP

Interpretation: UP, HS, MSP, JSV, and HJP

Funding acquisition: HJP

Investigation: UP, HS, JSV, and HJP

Methodology: UP and HJP

Project Administration: HJP

Supervision: HJP

Validation: UP, HS, and HJP

Visualization: UP, AN, TM, and HJP

Manuscript Preparation: UP, AN, TM, HS, JSV, and HJP

\section{ACKNOWLEDGEMENT}

Uttam M. Pal acknowledges Dr. Vineet Dravid for helping in data analysis. Uttam M. Pal also acknowledges Ms. Arpita Sarkar and Saeed Rila for helping with illustrations in Fig. 1-2 in the manuscript. Hardik J. Pandya acknowledges Tata Trusts for the short-term travel grant which resulted in collaboration with Prof. Jayant S. Vaidya, University College London.

\section{REFERENCES}

[1] U. M. Pal et al., "Optical spectroscopy-based imaging techniques for the diagnosis of breast cancer: A novel approach," Applied Spectroscopy Reviews. Taylor and Francis Inc., Sep. 13, 2020.

[2] U. M. Pal et al., "Towards a Portable Platform Integrated with Multispectral Noncontact Probes for Delineating Normal and Breast Cancer Tissue Based on Near-Infrared Spectroscopy," IEEE Trans. Biomed. Circuits Syst., vol. 14, no. 4, pp. 879-888, 2020.

[3] T. Nowikiewicz et al., "Clinical outcomes of an intraoperative surgical margin assessment using the fresh frozen section method in patients with invasive breast cancer undergoing breast-conserving surgery - a single center analysis," Sci. Rep., vol. 9, no. 1, pp. 1-8,
[4] M. C. Smitt et al., "The importance of the lumpectomy surgical margin status in long term results of breast conservation," Cancer, vol. 76, no. 2, pp. 259-267, 1995.

[5] J. S. Vaidya et al., "Long term survival and local control outcomes from single dose targeted intraoperative radiotherapy during lumpectomy (TARGIT-IORT) for early breast cancer: TARGIT-A randomised clinical trial," BMJ, vol. 370, 2020.

[6] M. Schnell et al., "All-digital histopathology by infrared-optical hybrid microscopy," Proc. Natl. Acad. Sci. U. S. A., vol. 117, no. 7, pp. 3388-3396, Feb. 2020.

[7] D. Grosenick et al., "Review of optical breast imaging and spectroscopy," J. Biomed. Opt., vol. 21, no. 9, p. 091311, Jul. 2016.

[8] P. G. Anderson et al., "Optical mammography: bilateral breast symmetry in hemoglobin saturation maps," J. Biomed. Opt., vol. 21, no. 10, p. 101403, 2016.

[9] S. L. Jacques, "Optical properties of biological tissues: A review," Physics in Medicine and Biology, vol. 58, no. 11. Jun. 07, 2013.

[10] J. Depciuch et al., "Comparing paraffined and deparaffinized breast cancer tissue samples and an analysis of Raman spectroscopy and infrared methods," Infrared Phys. Technol., vol. 76, no. May 2018, pp. 217-226, 2016

[11] J. Depciuch et al., "Monitoring breast cancer treatment using a Fourier transform infrared spectroscopy-based computational model," J. Pharm. Biomed. Anal., vol. 143, pp. 261-268, Sep. 2017.

[12] D. C. Fernandez et al., "Infrared spectroscopic imaging for histopathologic recognition," Nat. Biotechnol., vol. 23, no. 4, pp. 469-474, 2005.

[13] P. Anastasiadis et al., "High-Frequency Time-Resolved Scanning Acoustic Microscopy for Biomedical Applications," Open Neuroimag. J., vol. 12, no. 1, pp. 69-85, Jan. 2019.

[14] L. Ruby et al., "Breast Cancer Assessment With Pulse-Echo Speed of Sound Ultrasound From Intrinsic Tissue Reflections," Invest. Radiol., vol. 54, no. 7, pp. 419-427, Jul. 2019.

[15] H. J. Pandya et al., "Toward a Portable Cancer Diagnostic Tool Using a Disposable MEMS-Based Biochip," IEEE Trans. Biomed. Eng., vol. 63, no. 7, pp. 1347-1353, Jul. 2016.

[16] H. J. Pandya et al., "Design and fabrication of a flexible MEMSbased electro-mechanical sensor array for breast cancer diagnosis," J. Micromechanics Microengineering, vol. 25, no. 7, Jul. 2015.

[17] H. J. Pandya et al., "Mechanical phenotyping of breast cancer using MEMS: A method to demarcate benign and cancerous breast tissues," Lab Chip, vol. 14, no. 23, pp. 4523-4532, Dec. 2014.

[18] H. J. Pandya et al., "Towards an automated MEMS-based characterization of benign and cancerous breast tissue using bioimpedance measurements," Sensors Actuators, B Chem., vol. 199, pp. 259-268, 2014.

[19] M. R. Casu et al., "A COTS-Based Microwave Imaging System for Breast-Cancer Detection," IEEE Trans. Biomed. Circuits Syst., vol. 11, no. 4, pp. 804-814, Aug. 2017.

[20] A. Santorelli et al., "A Time-Domain Microwave System for Breast Cancer Detection Using a Flexible Circuit Board," IEEE Trans. Instrum. Meas., vol. 64, no. 11, pp. 2986-2994, Nov. 2015.

[21] H. Choi et al., "Development of a multiwavelength visible-rangesupported opto-ultrasound instrument using a light-emitting diode and ultrasound transducer," Sensors (Switzerland), vol. 18, no. 10, 2018.

[22] G. Yuan et al., "Light-emitting diode-based multiwavelength diffuse optical tomography system guided by ultrasound," J. Biomed. Opt., vol. 19, no. 12, p. 126003, 2014.

[23] S. W. Choe et al., "Combinational light emitting diode-high frequency focused ultrasound treatment for HeLa cell," Comput. Assist. Surg., vol. 22, no. 0, pp. 79-85, 2017.

[24] F. N. Ghartey et al., "Breast-i is an effective and reliable adjunct screening tool for detecting early tumour related angiogenesis of breast cancers in low resource sub-saharan countries," Int. J. Breast Cancer, vol. 2018, 2018.

[25] J. Min et al., "Computational Optics Enables Breast Cancer Profiling in Point-of-Care Settings," ACS Nano, vol. 12, no. 9, pp. 9081-9090, 2018.

[26] H. Vavadi et al., "Compact ultrasound-guided diffuse optical tomography system for breast cancer imaging," J. Biomed. Opt., vol. 24 , no. 02 , p. 1 , Oct. 2018.

[27] M. J. Baker et al., "Using Fourier transform IR spectroscopy to analyze biological materials," Nat. Protoc., vol. 9, no. 8, pp. 1771- 
1791, Jul. 2014

[28] H. J. Butler et al., "Shining a light on clinical spectroscopy: Translation of diagnostic IR, 2D-IR and Raman spectroscopy towards the clinic," Clin. Spectrosc., vol. 1, p. 100003, Dec. 2019.

[29] S. Kumar et al., "Role of Infrared Spectroscopy and Imaging in Cancer Diagnosis," Curr. Med. Chem., vol. 25, no. 9, pp. 10551072, 2018.

[30] W. Qiu et al., "A multifunctional, reconfigurable pulse generator for high-frequency ultrasound imaging," IEEE Trans. Ultrason. Ferroelectr. Freq. Control, vol. 59, no. 7, pp. 1558-1567, 2012.

[31] Y. Qin et al., "Optimizing frequency and pulse shape for ultrasound current source density imaging," IEEE Trans. Ultrason. Ferroelectr. Freq. Control, vol. 59, no. 10, pp. 2149-2155, 2012.

[32] K. W. Lin et al., "Synthesis of monopolar ultrasound pulses for therapy: The frequency-compounding transducer," IEEE Trans. Ultrason. Ferroelectr. Freq. Control, vol. 61, no. 7, pp. 1123-1136, 2014.

[33] M. Hauck et al., "Comparison of the effects of $1 \mathrm{MHz}$ and $3 \mathrm{MHz}$ therapeutic ultrasound on endothelium-dependent vasodilation of humans: a randomised clinical trial," Physiother. (United Kingdom), vol. 105, no. 1, pp. 120-125, 2019.

[34] K. K. Shung, Diagnostic Ultrasound. Boca Raton: CRC Press, 2015.

[35] P. Taroni et al., "Non-invasive optical estimate of tissue composition to differentiate malignant from benign breast lesions: A pilot study," Sci. Rep., vol. 7, no. January, pp. 1-12, 2017.

[36] S. K. V. Sekar et al., "Diffuse optical characterization of collagen absorption from 500 to $1700 \mathrm{~nm}$," J. Biomed. Opt., vol. 22, no. 1, p. 015006, 2017.

[37] S. A. Prahl, "Everything I think you should know about inverse adding-doubling," Oregon Medical Laser Center, St. Vincent Hospital, 2011. http://omlc.ogi.edu/software/iad.

[38] J. Cen et al., "Double-integrating-sphere system for measuring the optical properties of tissue based on single-chip microcomputer," $Y i$ Qi Yi Biao Xue Bao/Chinese J. Sci. Instrum., vol. 25, no. 2, p. 187, 2004.

[39] J. R. Cook et al., "Tissue-mimicking phantoms for photoacoustic and ultrasonic imaging," Biomed. Opt. Express, vol. 2, no. 11, p. 3193, 2011.

[40] G. Liu et al., "Fabrication of a multilayer tissue-mimicking phantom with tunable optical properties to simulate vascular oxygenation and perfusion for optical imaging technology," Appl. Opt., vol. 57, no. 23, p. 6772, 2018

[41] P. D. Cook et al., "Prediction of tissue optical properties using the Monte Carlo modeling of photon transport in turbid media and integrating spheres," OSA Contin., vol. 3, no. 6, p. 1456, 2020.

[42] L. B. da Cruz Junior et al., "Manufacture and characterization of a 3D-printed integrating sphere," Instrum. Sci. Technol., vol. 0, no. 0 , pp. 1-12, 2020.

[43] B. L. Sprague et al., "Variation in Mammographic Breast Density Assessments Among Radiologists in Clinical Practice: A Multicenter Observational Study.," Ann. Intern. Med., vol. 165, no. 7, pp. 457-464, Oct. 2016.

[44] E. White et al., "Variation in mammographic breast density by time in menstrual cycle among women aged 40-49 years.," J. Natl. Cancer Inst., vol. 90, no. 12, pp. 906-10, Jun. 1998.

[45] S. Azam et al., "Mammographic Density Change and Risk of Breast Cancer," J. Natl. Cancer Inst., vol. 112, no. 4, pp. 391-399, Apr. 2020.

[46] N. F. Boyd et al., "Mammographic densities and breast cancer risk.," Cancer Epidemiol. Prev. Biomarkers, vol. 7, no. 12, 1998

[47] E. Y. Kim et al., "Mammographic breast density, its changes, and breast cancer risk in premenopausal and postmenopausal women," Cancer, vol. 126, no. 21, pp. 4687-4696, Nov. 2020.

[48] O. Abdel Alim et al., "Modeling the acoustic attenuation process of soft tissues," J. Phys., vol. 4, no. 5 pt 2, pp. 1263-1266, 1994.

[49] N. Duric et al., "Detection of breast cancer with ultrasound tomography: First results with the Computed Ultrasound Risk Evaluation (CURE) prototype," Med. Phys., vol. 34, no. 2, pp. $773-$ 785, 2007.

[50] A. Irshad et al., "Assessing the role of ultrasound in predicting the biological behavior of breast cancer," Am. J. Roentgenol., vol. 200, no. 2, pp. 284-290, 2013.

[51] N. Yoshizawa et al., "Factors affecting measurement of optic parameters by time-resolved near-infrared spectroscopy in breast cancer," J. Biomed. Opt., vol. 23, no. 02, p. 1, Feb. 2018.

[52] Q. Fang et al., "Combined optical and x-ray tomosynthesis breast imaging," Radiology, vol. 258, no. 1, pp. 89-97, Jan. 2011.

[53] D. Grosenick et al., "Time-domain scanning optical mammography: II. Optical properties and tissue parameters of 87 carcinomas," Phys. Med. Biol., vol. 50, no. 11, pp. 2451-2468, Jun. 2005.

[54] R. Choe et al., "Differentiation of benign and malignant breast tumors by in-vivo three-dimensional parallel-plate diffuse optical tomography," J. Biomed. Opt., vol. 14, no. 2, p. 024020, 2009.

[55] Y. Yamada et al., "Time-domain near-infrared spectroscopy and imaging: A review," Applied Sciences (Switzerland), vol. 9, no. 6. MDPI AG, p. 1127, Mar. 17, 2019.

[56] P. G. Anderson et al., "Broadband optical mammography: Chromophore concentration and hemoglobin saturation contrast in breast cancer," PLoS One, vol. 10, no. 3, pp. 1-23, 2015. 